\title{
Research on the Integration and Utilization of Personal Non-tangible Idle Resources under Sharing Economy
}

\author{
Xin Chu ${ }^{1, \text { a }}$, Siyuan Li ${ }^{1, \text { a }}$, Shuai Zhou ${ }^{2, \text { a }}$ \\ ${ }^{1}$ Wuhan Donghu University, Wuhan, Hubei, 430212 \\ ${ }^{2}$ China University of Geosciences, Wuhan, Hubei, 430074 \\ aemail:chuxin0527@sohu.com
}

Keywords: Sharing economy, non-tangible idle resources, platform construction

\begin{abstract}
With the vigorous development of the "Internet +" economy, the "shared economy" has entered people's lives as a new thing, and has been deepening, developing, maturing and stable. Individual non-physical idle resources refer to individual resources that are idle in time, space, physical strength, and brain power, which are important components of social resources. If it can be effectively integrated and utilized, it will help to improve the use efficiency of individual resources, realize the rational allocation of idle resources, solve certain contradictions between supply and demand, and bring convenience to individual life and social operation. Therefore, under the wave of sharing economy, how to realize the effective use of personal non-tangible idle resources and how to construct relevant platforms deserves people's current research.
\end{abstract}

\section{Introduction}

"Sharing Economy", first published in 1978 by Marcus Felson, a sociology professor at Texas State University, and Joel Spaeth, a professor of sociology at the University of Illinois (Community) Structure and Collaborative Consumption: A Routine Activity Approach) means that an institution or individual with idle resources pays the right to use the resources to others, the transferor obtains a return, and the sharer uses the idle resources of others to create value. In just a few decades, the sharing economy has become an important force driving economic development, and has developed rapidly in the areas of accommodation, transportation, education, tourism, and life services. Excellent companies with the concept of sharing economy continue to emerge, sharing resources from pet fostering and sharing, parking space sharing to expert sharing, guide sharing, and various new models emerge one after another. At present, the sharing economy is developing in a direction of deepening, maturity and stability. In China's "13th Five-Year Plan", the sharing economy has risen to the national strategic level. It is affecting and changing the traditional business model. More resources and services will be concentrated in the shared platform in the future.

In practice, the sharing economy model presents the following characteristics: First, it is an Internet-based information technology-based exchange platform created by a third party. Individuals use these exchange platforms to exchange idle items, including tangible or intangible products, physical or intellectual, labor, knowledge or experience. Second, the sharing economy involves three main players, namely the demand side, the supply side and the sharing platform. Third, the sharing platform is used as a link between the supply and demand sides, and is established through a series of mechanisms such as mobile LBS application, dynamic algorithm and pricing, and mutual evaluation system, so that both the supply and demand sides can conduct transactions through the shared platform.

In the tide of the sharing economy, the integration and trading behavior of personal non-tangible idle resources become more and more common, and the sharing trend is becoming more and more obvious, and the importance is more prominent. Considering that individuals as social individuals, on the one hand, they cannot guarantee the validity and continuity of idle resource sharing on their own. On the other hand, individuals are the holders of private resources and the end of the resource chain. Therefore, effective mobilization and integration It is of great significance to promote the 
sharing and co-governance of resources and the effective use of resources to achieve the sharing and co-processing of resources.

This paper, based on the research of the status quo of the use of non-tangible idle resources in the context of the sharing economy, analyzes the feasibility and countermeasures of the integrated use and countermeasures, and proposes the sharing of the use of non-tangible idle resources. A new economic practice model, with a view to maximizing the utilization of related resources and achieving rational and effective allocation of resources.

\section{Basic concepts and main features of personal non-tangible idle resources}

Personal non-tangible idle resources refer to the intangible resources of the individual's personal time, space, physical strength, and mental power remaining after satisfying their own life or work needs. The owner of the resource tries to maximize the use of his idle resources and help the resource demanders achieve their life or work goals by means of transfer, rental, and sale. In this regard, the difference between the highest price that resource demanders are willing to pay and the actual market price of these non-tangible idle resources is the market value of the individual's non-tangible idle resources. Of course, this should be based on an equal and voluntary contract between the two parties. In the above way, in the case where the resources available to the society are relatively scarce, one party is willing to pay at a price that is relatively flat or higher than the price of the service market based on the contractual relationship; the other party actively supplies idle resources and promotes the completion of the transaction, ultimately making The idle social resources have been exchanged, generated value, and promoted the effective use of resources.

For most people, the rational allocation of time and the efficient use of resources can simplify their lives. People often encounter problems of uneven distribution in the distribution and use of their own production and living materials, which leads to serious polarization of time and efficiency. When people are busy, there will be a large number of production and life tasks that cannot be balanced, cannot be completed efficiently, and generate demand for non-tangible resources of others; on the contrary, individuals will produce excess or idleness of non-tangible resources at different times. Non-tangible idle resources are essentially a potential social and time value. Insufficient use will inevitably lead to waste of resources and low efficiency of social production. Faced with the gap between demand and waste, it is the inevitable social development to make full use of individual non-tangible idle resources, stimulate the use potential of resources, and improve the utilization rate of resources so that both the supply and demand sides of resources can benefit from it.

The author found through investigation that 186 of the 228 respondents had the intention of selling non-tangible idle resources, and 32\% of the respondents were "very interested"; among them, they have used such tasks as "tasks". The number of people who have idle resources sharing platforms is 29 , and the number of people who have paid for practice accounts for nearly $16 \%$ of the number of people who are willing to pay; 179 people have the idea of buying or renting idle resources for others. Among them, about 30\% of respondents have strong intentions. Among the groups that are "positively optimistic about the behavior of individuals with non-tangible idle resources", young people aged 18 to 22 are the mainstay, accounting for nearly $60 \%$ of the total. The above data show that personal non-tangible idle resources have not been effectively and fully developed and utilized in the current practice, but in reality, their needs exist objectively. It can be seen that shared resources have certain market prospects.

In the current situation of sharing the economy, there are many cases of effective use of non-physical and idle resources. The most typical ones are "Drips and Windmills". Despite the recent infringement and infringement of the piggybacking people, the development of "winding" has been affected and restricted. However, from the perspective of economic development, the development of "shunning" is still the inevitable development of the current sharing economy. The product is an unstoppable economic trend. We must be soberly aware that the occurrence of various incidents of people carrying people is only an individual event that is difficult to avoid in the current sharing economy operation, development and improvement process. It does not negate this 
sharing model. "Shunting" is still a typical form of promoting the use of personal goods in the private sector, and is one of the examples of the effective use of non-tangible idle resources.

In terms of characteristics, personal non-tangible idle resources have the advantages of flexibility, convenience, and economy, and also have shortcomings such as uncontrollability, insecurity and complexity. At the same time, due to the limitations of personal data processing and analysis capabilities, individuals' ability to integrate and utilize resources is very limited. Therefore, it is becoming more and more important to build a platform that can effectively integrate personal idle resources.

\section{The current situation and problems of the use of personal idle resources}

Integrating personal non-tangible idle resources, in essence, is more about the sharing of non-tangible assets. It is different from the shared form of tangible assets of Mobai and ofo shared bicycles, but it also has the flexibility of tangible asset sharing. Convenience, reward and other characteristics. In practice, resource suppliers make full use of the characteristics of individual resource flexibility, and strive to improve the use efficiency of personal time, space, physical strength, mental resources and other resources, and can meet the needs of others when completing their own tasks, reflecting convenience, Flexibility and sharing. At the same time, the sharing party obtains the rewards of others and also reflects the return.

Although sharing is the inevitable development, as a new thing, personal non-tangible idle resources also have many problems in the current sharing process. First of all, from the perspective of the existence status of the resource itself, the individual idle resource owner provides the idle resource information to the resource demander through the individual or the platform. At this time, the supply and demand sides are based on the contractual relationship of the willingness of the two parties to cooperate, and the two parties establish a de facto Practical contracts, the way in which resources can be provided and the way in which resources are provided, time, place, etc. are often determined by the individual idle resource owner, and the other party cannot enforce the requirement. This mode of benefiting the individual idle resource owner will lead to binding. It is weak, uncontrollable, and cannot guarantee a stable supply of resources.

Secondly, although many shared platforms require users to ensure the authenticity and effectiveness of their identity through real-name authentication, the highly open features of the shared platform determine that users can minimize costs and enter the platform. At the same time, the platform cannot The user conducts systematic training and management, and even cannot implement a strict auditing system, which leads to insecurity. The typical case is the flight attendant incident in the "winding".

Thirdly, compared with the traditional economic form, the sharing economy is based on the Internet as a communication and trading medium, which makes the structure of its social relationship complicated, especially in the context of the existing laws and regulations are still under construction and perfection. When the rights of the two parties are damaged and various disputes occur, the definition of risk, the division of responsibilities, and the maintenance of rights and interests are insufficient in terms of hard binding, which restricts the development of resource sharing.

\section{The economic model of the use of personal non-tangible idle resources}

Facing the objective needs of personal non-tangible idle resource sharing, huge market, considerable benefits, and problems exposed in practice, shortcomings and shortcomings, building a reasonable, efficient and secure resource sharing platform is undoubtedly one of the important ways to solve the problem.

In contrast, the traditional market economy often uses big customer transactions as the core of enterprise competition. Conversely, the sharing economy dominates precisely the "fine market" that is neglected by the traditional market economy. For example, the service provided by the "last mile" is a fine market that traditional transportation is unwilling to enter. The emergence of shared 
bicycles breaks the shackles of regional and traditional transportation and realizes the "last mile" demand of people. Therefore, the construction of the personal non-tangible idle resource trading platform is aimed at solving the "last mile" problem when the individual idle resources are traded, to meet the relevant needs, and has the practical importance.

Technically, the emergence of the Internet has brought great convenience to people's production and life. In this context, the sharing economy can provide key media, interaction and information processing functions for both the supply and demand sides through the Internet's big data processing capabilities. The effective conversion of idle resources into social resources, and ultimately the efficient allocation of personal non-tangible idle resources, makes the construction of personal non-tangible idle resource trading platform technically feasible.

In the business model, in the wave of the Internet and the sharing economy, the most suitable for the integration and utilization of personal non-tangible idle resources is undoubtedly $\mathrm{O} 2 \mathrm{O}$ mode and C2C mode. In fact, in the application of this field, there is actually no obvious boundary between the two. Whether it is the popular "Drips" or "Tasks", it is developed on the basis of these two modes. In practice, online malls and service websites established according to the $\mathrm{O} 2 \mathrm{O}$ model are promoted through online network marketing; online social media interaction with customers is established through $\mathrm{C} 2 \mathrm{C}$, and an online and offline integrated marketing system is established, through e-commerce behavior among consumers. Seal the deal. This makes the trading platform operational in practice and has accumulated valuable real-world experience.

In general, the resource sharing platform, as a core node and transaction medium that connects the supply and demand of personal non-tangible idle resources, can not only bring convenience to the owners and demanders of idle resources, but also through the processing of big data on the Internet. Overcome the limitation of information asymmetry, realize the organic integration of supply, demand, configuration, sharing and co-governance, and promote the rational and effective allocation of personal non-tangible idle resources. Of course, in addition to the construction of shared platforms, promoting the development of the sharing economy and promoting the re-allocation and reuse of non-tangible idle resources, it is necessary for the government and the society to actively cooperate with each other, based on the characteristics of the sharing economy, operational rules, and risk distribution. Improve the management and control system, re-examine the adaptability of relevant legal systems, give more legal protection to resources, and further establish and improve the industry normative system, and build a multi-layered combination of autonomous supervision, platform supervision, government supervision and legal supervision. Management system.

General Secretary $\mathrm{Xi}$ pointed out in the report of the 19th National Congress that resource integration is an important guarantee for the continued development of social services. As a new resource integration entry point, the "shared economy" model may change the dilemma of social resources in our country and gradually change the way social services are supplied. At present, the sharing economy has risen to the national strategic level. It not only reconstructs the business model, but also promotes the transformation of the traditional economy, and brings more and new opportunities and challenges. In this wave, the shared platform will focus on more existing resources. Further construction and full use of relevant platforms can effectively integrate, configure and utilize personal non-tangible idle resources, reduce transaction costs, improve resource use efficiency, and increase total social supply. At the same time, its congenital "mutual help" and "public welfare" genes will improve the social relationship between people, change people's life and consumption behavior to a certain extent, and promote service convenience, resource conservation, and life science and technology. The formation of a business model that facilitates people's lives and benefits the whole society.

\section{Acknowledgements}

Fund Project: The project was supported by the 2017 National Undergraduate Innovation and Entrepreneurship Training Program “Interchange? Student Task Interchange Project” approved by 
Wuhan Donghu University, project number 201711798002.

\section{References}

[1] Marcus Felson, Joel Spaeth. Community Structure and Collaborative Consumption: A Routine Activity Approach [J], American Behavioral Scientist, 1986, 21 (4): 614-624.

[2] Wu Chaoya, Promoting the Resource Sharing and Development of Universities with the Network Development of Resource Platform[J], China Adult Education, 2016, 06, 41-43.

[3] Li Fengzhi, Suo Wei, Zhu Yun, Liu Yu, Carrying out the spirit of national scientific and technological innovation planning in the "13th Five-Year Plan", strengthening the open sharing of science and technology resources and the construction of service platforms [J], Scientific Management Research, 2017, 05, 22-25.

[4] Liu Pei, Construction of Open Information Resource Sharing Platform in Big Data Environment [J], Electronic Technology and Software Engineering, 2018, 18, 151. 